Please do not remove this page

RMIT

UNIVERSITY

\title{
Adaptive receiver beamforming for diversity coded OFDM systems: Maximum SNR design
}

Lin, Kevin; Hussain, Zahir

https://researchrepository.rmit.edu.au/esploro/outputs/9921858797601341/filesAndLinks?institution=61RMIT_INST\&index=null

Lin, K., \& Hussain, Z. (2005). Adaptive receiver beamforming for diversity coded OFDM systems: Maximum SNR design. Proceedings of the Tencon 2005 IEEE Region 10 Conference.

https://doi.org/10.1109/TENCON.2005.301115

Published Version: https://doi.org/10.1109/TENCON.2005.301115

Repository homepage: https://researchrepository.rmit.edu.au

(c) 2005 IEEE. Personal use of this material is permitted. However, permission to reprint/republish this material for advertising or promotional purposes or for creating new collective works for resale or redistribution to servers or lists, or to reuse any copyrighted component of this work in other works must be obtained from the IEEE.

Downloaded On 2023/04/26 22:42:51 +1000 


\title{
Adaptive Receiver Beamforming for Diversity Coded OFDM Systems: Maximum SNR Design
}

\author{
Kevin H. Lin and Zahir M. Hussain \\ School of Electrical and Computer Engineering, RMIT University, Melbourne, Australia \\ Emails: s9510490@student.rmit.edu.au; zmhussain@iee.org
}

\begin{abstract}
Over the recent years, advance multiantenna transmission schemes have attracted considerable interest due to their potential benefits in improving the system capacity and error-rate performance. As a result, space-time coding and transmit beamforming have emerged as the two most promising techniques. Because of limited space at the mobile station (MS) and the fact that download intensive services are to be introduced in the next generation of cellular systems, most of research efforts have been pouring on transmit diversity techniques. In this paper, we focus on adaptive uplink transmission and reception techniques for wireless communications and introduce a new frequency-time encoding scheme that can be used to exploit frequency diversity branches for broadband OFDM systems with only one antenna at the MS. By incorporating this with receive beamforming at the base station (BS), the instantaneous signalto-noise ratio (SNR) is maximized and the system error-rate performance is then further improved. Numerical results showed that systems employed the proposed transceiver structure have a 4-dB improvement over the conventional space-time coding scheme when two receive antennas are used.
\end{abstract}

\section{INTRODUCTION}

Signal transmission in multi-input multi-output (MIMO) systems that employs more than one antennas at the transmitter and the receiver has shown to be effective in exploiting spatial diversified paths of wireless channels [1]-[2] and increasing both system capacity and error-rate performance. In particular, space-time coding includes both space-time block coding [3]-[4] and trellis coding [5] had gained a significant attention due to their superior performance and simplicity of transceiver design over other known techniques. However, their performance improvements are based on the assumption that the arriving multipath signals are sufficiently uncorrelated. In cellular communications, due to close spacing between antenna elements at the base station (BS), signal paths are often correlated to some degree. As a consequence, coherent deep fade between propagation signal paths is unavoidable and studies have shown that signal correlation can degrade the system performance significantly [6], [7].

The application of space-time coding to orthogonal frequency division multiplexing (OFDM) systems was first introduced in [8]. Motivated by the presence of additional multipath diversity offered by frequency-selectivity in broadband wireless channels, space-frequency (SF) [7] and space-timefrequency (STF) coding [9] were introduced. Extending from their work, the combination of diversity coding schemes with transmit beamforming was investigated for broadband OFDM systems [10]-[11]. However, all of these transmission schemes are for downlink application with multiple transmit antennas.

In this paper we propose a new frequency-time (FT) encoding scheme and combine it with receiver beamforming (denote by FT-Beam) to maximize the received signal-to-noise (SNR) for OFDM systems with single transmit antenna. By knowing subchannel gains at the mobile station (MS), we utilize the concept of subchannel grouping in [12] and perform FT encoding of existing space-time codes across OFDM subcarriers to achieve transmit diversity in the frequency domain. With the effective use of beamforming at the multiantennas base station (BS) receiver, the optimal adaptive beam-mapping weights is applied to maximize the instantaneous SNR, and thus, system error-rate performance during uplink transmission in a singleinput multi-output (SIMO) channel is further enhanced.

Notation used: $(\cdot)^{*},(\cdot)^{T}$, and $(\cdot)^{H}$ are complex conjugate, vector transposition, and Hermitian transposition, respectively. $\|\cdot\|_{F}$ is the Frobenius norm; $\sqrt{\mathcal{A}}$ stands for Hermitian square root of matrix $\mathcal{A}$; $\operatorname{det}(\cdot)$ denotes the determinant; $\mathcal{E}\{\cdot\}$ is the expectation operator. Finally, capital (small) bold letters represent matrices (vectors).

\section{SySTEM MOdEL}

Consider an uplink cellular communication scenario employing an $N_{c}$ frequency tone OFDM system with a single transmit antenna at the MS and $N_{r}$ receive antennas at the BS over a frequency-selective fading channel. It is assumed that the channel coherent bandwidth is larger than the bandwidth of each subcharrier; we thus consider the corresponding subchannel to be frequency-flat. In Fig. 1, we depicted a general structure of this OFDM system and combined with the proposed adaptive transceiver structure. In this work, it is also assumed that the system operates in a typical cellular environment where the BS antennas are placed at the building roof-top in an unobstructed manner. It is stated in [13] that signal transmission in such an environment over a multipath channel will lead to partially correlated signal paths in the spatial domain arriving at the BS. Next, assume that a uniform linear array (ULA) configuration is used for $N_{r}$ BS antennas with a spacing of $d$ meters. The normalized correlation matrix that specifies the correlation between antenna elements is 


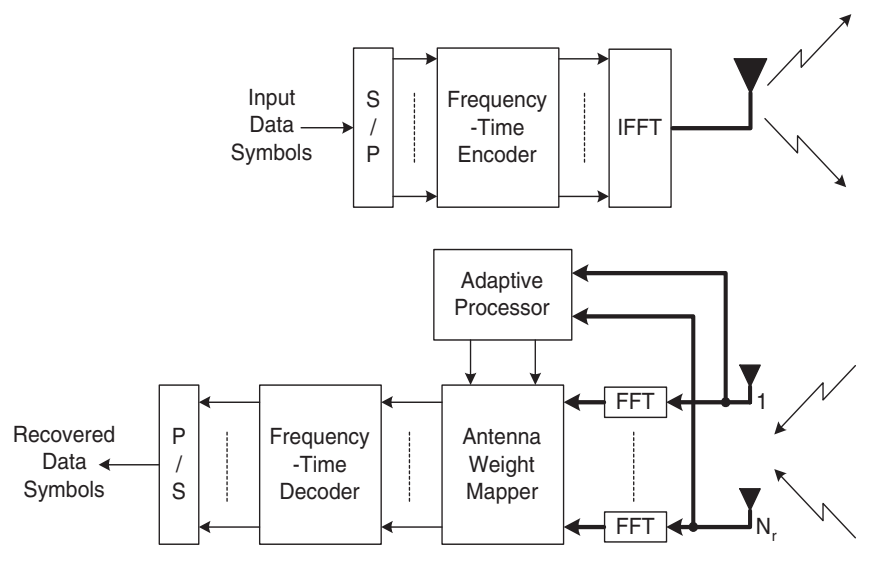

Fig. 1. General structure of FT-Beam OFDM system with adaptive receive-beamforming. Bold arrows represent multi-line signals.

defined in [14] as

$$
\mathbf{R}_{r}=\frac{1}{L} \sum_{\ell=1}^{L} \mathbf{a}\left(\theta_{\ell}\right) \mathbf{a}^{H}\left(\theta_{\ell}\right)
$$

where $L$ denotes the number of dominant resolvable paths and $\mathbf{a}\left(\theta_{\ell}\right):=\left[1, e^{j \beta}, e^{j 2 \beta}, \cdots, e^{j\left(N_{r}-1\right) \beta}\right]^{T}$ is the array propagation vector for the $\ell$ th tap with an angle-of-arrival (AoA) of $\theta_{\ell} \cdot \beta=\left[2 \pi \cdot d \cdot \sin \left(\theta_{\ell}\right)\right] / \lambda, \lambda$ being the carrier frequency wavelength. In general, $\mathbf{R}_{r}$ is a nonnegative-definite Hermitian matrix and the eigenvalue-decomposition (EVD) of $\mathbf{R}_{r}$ can be expressed as $\mathbf{V R}_{r} \mathbf{V}^{H}=\boldsymbol{\Delta}$, where $\mathbf{V}=\left[\boldsymbol{v}_{1}, \cdots, \boldsymbol{v}_{N_{t}}\right]$ is a unitary matrix with columns that are the eigenvectors and $\boldsymbol{\Delta}=\operatorname{diag}\left[\delta_{1}, \cdots, \delta_{n}, \cdots, \delta_{N_{r}}\right]$ is a diagonal matrix contains the corresponding eigenvalues. Without loss of generality, we assume that $\delta_{n}$ 's are ordered in a non-increasing fashion: $\delta_{1} \geq \delta_{2} \geq \cdots \geq \delta_{N_{r}} \geq 0$.

Let us denote the correlated SIMO channel frequency response vector for the $k^{\text {th }}$ subcarrier as $\mathbf{h}_{k} \in \mathbb{C}^{1 \times N_{r}}$. The $j^{\text {th }}$ element, which represents the subchannel gain between the transmit and the $j^{\text {th }}$ receive antenna, is defined as $h_{k}(j):=$ $\mathbf{g}_{j} \mathbf{f}_{k}$, where $\mathbf{g}_{j}=\left[g_{j}(1), \cdots, g_{j}(L-1)\right]$ is the channel impulse response vector with independent circularly symmetric complex Gaussian random variables from $\mathcal{C N}\left(0, \sigma_{h}^{2}\right)$ and $\mathbf{f}_{k}=\left[1, e^{-j 2 \pi(k-1) / N_{c}}, \cdots, e^{-j 2 \pi(k-1) \tau_{L-1} / N_{c}}\right]^{T}$ is the corresponding discrete Fourier transform coefficients. According to [13], the channel frequency response vector can also be expressed as $\mathbf{h}_{k}=\overline{\mathbf{h}}_{k} \sqrt{\mathbf{R}_{r}}$, where $\overline{\mathbf{h}}_{k}$ can be thought as a prewhitened channel vector. Furthermore, quasi-static fading is also assumed throughout the duration of one FTBC codeword length but fading may vary from one block to another.

\section{A. Subchannel Grouping \& Frequency-Time Encoding}

The concept of subchannel grouping, sometimes referred as subcarrier grouping, was originally used in adaptive modulation scheme in [10] to reduce processing complexity by grouping subcarriers or subchannels that are within one channel coherent bandwidth and having a similar fading gain.

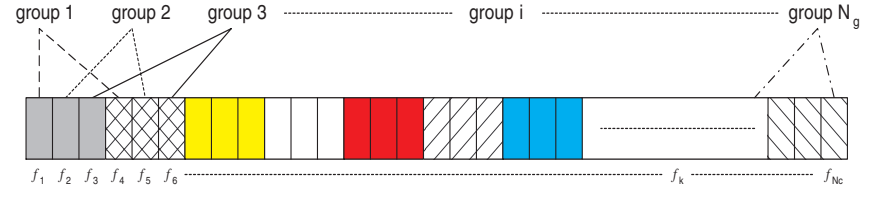

Fig. 2. An illustration of subchannel grouping for OFDM systems with $N_{c}$ frequency tones and the channel coherent bandwidth is equivalent to the bandwidth of 3 subcarriers.

In [9] and [12], subcarrier grouping is used for grouping frequencies that are approximately one coherent bandwidth apart to perform STF coding in OFDM systems to exploit both spatial and multipath diversity. In this work, we utilize the concept of subchannel grouping in [12] by treating subcarriers that are having different fading gains as additional antennas at the MS. By doing so, we can then directly apply the spacetime codes in [3]-[5], [15] in our system by spreading symbol energy across OFDM frequencies instead of antennas. An illustratation of this subchannel grouping concept is shown in Fig. 2, where the channel coherent bandwidth is assumed to be equivalent to three frequency tones and subchannels that are having different fading gains are grouped together. Thus, the number of subchannels (subcarriers) that are in one group depends on the spatial dimension of the original space-time code.

Let us denote $N_{g}$ as the total number of groups as a result of this sub-channel grouping process. If the well-known Alamouti's space-time block code in [3]

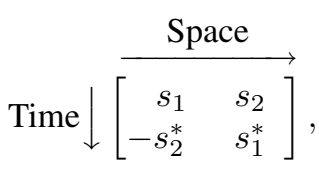

(which has a spatial dimension of 2) is used for the FT encoding process, then $N_{g}=N_{c} / 2$. An example of this FT encoding output is shown in Fig. 3, where baseband modulated data symbols $s_{1}$ and $s_{2}$ are FT-encoded across two subcarriers $f_{1}$ and $f_{4}$ (c.f. Fig. 2) as well as two OFDM symbol periods $n=0$ and $n=1$ ( $n$ being the time index in mod-2 sense). Similarly, data symbols $s_{3}$ and $s_{4}$ are encoded in group 2, while $s_{5}$ and $s_{6}$ are encoded in group 3. Note that other STBC matrices in [4] and STTC technique in [5] can be applied in the same way, but with different subchannel grouping sizes.

\section{B. Receiver Beamforming}

At the receiver, discrete Fourier transformation is applied to the noisy samples of SIMO signals arriving at $N_{r}$ antennas. Assume ideal symbol-time sampling and carrier synchronization, the discrete time baseband equivalent form of system input-output equation can be expressed as

$$
\mathbf{r}_{k}=x_{k} \overline{\mathbf{h}}_{k} \sqrt{\mathbf{R}_{r}}+\mathbf{e}_{k},
$$

where $x_{k}$ is an FT encoded data symbol transmitted on the $k^{\text {th }}$ subcarrier, and $\mathbf{e}_{k}$ is a additive white Gaussian noise vector with each element having zero mean and $\sigma_{k}^{2}$ variance. 


\section{Timing index, $n$}

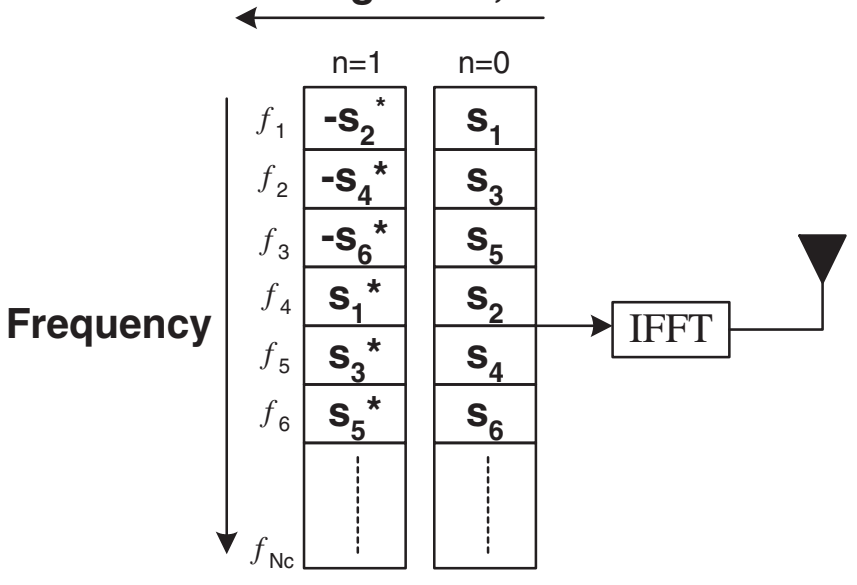

Fig. 3. Proposed FT encoding of Alamouti's space-time block code with only one transmit antenna.

Estimation of the channel fading gains is carried out at the adaptive processor (AP) by correlating pilot tones embedded in the transmitted signal. Results are then used for generating the antenna weighting matrix to maximize the received SNR. The antenna weight mapping process is performed across $N_{r}$ receive antennas. Mathematically, it can be written as

$$
\mathbf{y}_{k}=\mathbf{r}_{k} \mathbf{W}_{k}=x_{k} \overline{\mathbf{h}}_{k} \sqrt{\mathbf{R}_{r}} \mathbf{W}_{k}+\mathbf{e}_{k} \mathbf{W}_{k},
$$

where $\mathbf{W}_{k}={\sqrt{\mathbf{U}_{k} \mathbf{W}}}^{H}$ is the weighting matrix for the $k^{\text {th }}$ subcarrier, $\overline{\mathbf{W}}=\left[\overline{\mathbf{w}}_{1}, \cdots, \overline{\mathbf{w}}_{j}, \cdots, \overline{\mathbf{w}}_{N_{r}}\right]^{T} \in \mathbb{C}^{N_{r} \times N_{r}}$, and $\overline{\mathbf{w}}_{j}$ is the steering weight vector for the $j^{\text {th }}$ receive antenna. The matrix $\mathbf{U}_{k}=\operatorname{diag}\left[\mu_{1, k}^{2}, \cdots, \mu_{N_{r}, k}^{2}\right]$ contains the power splitting ratio for the steering weight vectors.

\section{Frequency-Time Decoding}

To recover original data, channel estimation results are also used for the FT decoding process. Since frequency subcarriers are treated as additional transmit antennas at the transmitter FT encoding, the decoding algorithm is divided into a combining stage and a maximum likelihood decoding (MLD) stage. The combining stage is simply adding the received signals in different subcarriers that are within the same subchannel group.

Denote $i(m)$ as the frequency index for subchannel group $i$. Using the previous example (c.r. Fig. $2 \& 3$ ), the frequency index of subchannel group 1 will read 1(1), which corresponds to subcarrier 1, and 1(2) corresponds to subcarrier 4. Thus, without the presumption that Alamouti's space-time block code is used for the FT encoding, the combining process can be expressed as

$$
\mathbf{y}_{i}(n)=\sum_{m=1}^{N_{c} / N_{g}} \mathbf{y}_{i(m)}(n)
$$

Following the maximum likelihood detection rule in [15], FT decoding of symbol $s_{1}$ of Alamouti's code amounts minimiz- ing the decision matrix in subchannel group $i$ as

$$
\begin{gathered}
\left|\left[\sum_{j=1}^{N_{r}}\left(y_{i, j}(n) h_{i(1)}^{*}(j)+y_{i, j}^{*}(n+1) h_{i(2)}(j)\right)\right]-s_{1}\right|^{2} \\
+\left(-1+\sum_{j=1}^{N_{r}} \sum_{m=1}^{2}\left|h_{i(m)}(j)\right|^{2}\right)\left|s_{1}\right|^{2},
\end{gathered}
$$

where $h_{i(m)}(j)$ and $y_{i, j}(n)$ denotes the $j^{\text {th }}$ entry of $\overline{\mathbf{h}}_{i(m)} \sqrt{\mathbf{R}_{r}} \mathbf{W}_{i(m)}$ and $\mathbf{y}_{i}(n)$, respectively. The decision matrix

$$
\begin{gathered}
\left|\left[\sum_{j=1}^{N_{r}}\left(y_{i, j}(n) h_{i(2)}^{*}(j)-y_{i, j}^{*}(n+1) h_{i(1)}(j)\right)\right]-s_{2}\right|^{2} \\
+\left(-1+\sum_{j=1}^{N_{r}} \sum_{m=1}^{2}\left|h_{i(m)}(j)\right|^{2}\right)\left|s_{2}\right|^{2},
\end{gathered}
$$

is used for decoding $s_{2}$. Similarly, the above MLD expressions can be easily extended and used for decoding of other spacetime block codes in [4].

\section{Signal-to-Noise Ratio (SNR)}

Given that the average transmitted energy during one OFDM-symbol interval is

$$
\mathcal{E}\left\{x_{k}\right\}=\mathcal{E}\left\{\left|s_{n}\right|^{2}\right\}=\varepsilon_{s},
$$

the received SNR at the $k^{\text {th }}$ subcarrier for the detection of $x_{k}$ has the form:

$$
\gamma_{k}=\frac{\varepsilon_{s}\left\|\overline{\mathbf{h}}_{k} \sqrt{\mathbf{R}_{r}} \mathbf{W}_{k}\right\|_{F}^{2}}{\sigma_{k}^{2}\left\|\mathbf{W}_{k}\right\|_{F}^{2}} .
$$

Denote $\left|s_{n}-\tilde{s}_{n}\right|$ as the minimum distance between the underlying constellation symbols, then the symbol energy for both QAM and PSK modulation schemes are given in [16] as

$$
\begin{array}{ll}
\varepsilon_{s}=\frac{(M-1)\left|s_{n}-\tilde{s}_{n}\right|_{\mathrm{QAM}}^{2}}{6}, & \text { for QAM } \\
\varepsilon_{s}=\frac{\left|s_{n}-\tilde{s}_{n}\right|_{\mathrm{PSK}}^{2}}{4 \sin ^{2}(\pi / M)}, & \text { for PSK. }
\end{array}
$$

\section{Optimal Antenna Weighting Matrix: Maximum SNR DESIGN}

The objective of this Section is to maximize the received SNR in order to improve the system error-rate performance. Effectively, this amounts choosing the weight mapping matrix that maximize (8) by solving the following cost function:

$$
\begin{aligned}
\max _{\mathbf{W}_{k}} J= & \operatorname{det}\left(\mathbf{I}_{N_{r}}+\frac{\varepsilon_{s}\left\|\overline{\mathbf{h}}_{k} \sqrt{\mathbf{R}_{r}} \mathbf{W}_{k}\right\|_{F}^{2}}{\sigma_{k}^{2}\left\|\mathbf{W}_{k}\right\|_{F}^{2}}\right) \\
= & \operatorname{det}\left(\mathbf{I}_{N_{r}}+\frac{\varepsilon_{s} \overline{\mathbf{h}}_{k} \sqrt{\mathbf{R}_{r}} \mathbf{W}_{k} \mathbf{W}_{k}^{H} \sqrt{\mathbf{R}_{r}}{ }^{H} \mathbf{h}_{k}^{H}}{\sigma_{k}^{2}\left\|\mathbf{W}_{k}\right\|_{F}^{2}}\right) \\
& \text { subject to : }\left\|\mathbf{W}_{k}\right\|_{F}^{2}=N_{r} .
\end{aligned}
$$

Equivalently, this can be re-written as

$$
\max _{\mathbf{W}_{k}} J=\operatorname{det}\left(\mathbf{I}_{N_{r}}+\frac{\varepsilon_{s} \overline{\mathbf{h}}_{k} \sqrt{\boldsymbol{\Lambda}} \boldsymbol{\Phi} \sqrt{\boldsymbol{\Lambda}}^{H} \overline{\mathbf{h}}_{k}^{H}}{N_{r} \sigma_{k}^{2}}\right),
$$


where $\boldsymbol{\Phi}_{k}=\mathbf{V}^{H}{\sqrt{\mathbf{U}_{k}}}^{H} \overline{\mathbf{W}}{\sqrt{\mathbf{U}_{k}}}^{H} \mathbf{V}$. By using Hadamard inequality, the optimization problem (12) can be rewritten as

$$
J \leq \operatorname{det}\left(\mathbf{I}_{N_{r}}+\frac{\varepsilon_{s} \overline{\mathbf{h}}_{k} \sqrt{\boldsymbol{\Lambda}} \boldsymbol{\Phi}_{k} \sqrt{\boldsymbol{\Lambda}}^{H} \overline{\mathbf{h}}_{k}^{H}}{N_{r} \sigma_{k}^{2}}\right)
$$

and the equality is achieved if and only if $\boldsymbol{\Phi}_{k}$ is a diagonal matrix. Assume $\boldsymbol{\Phi}_{k}=\operatorname{diag}\left[\phi_{1, k}, \phi_{2, k}, \cdots, \phi_{N_{r}, k}\right]$, hence (13) becomes

$$
J=\prod_{j=1}^{N_{r}}\left(1+\frac{\varepsilon_{s}}{N_{r} \sigma_{k}^{2}}\left|\bar{h}_{k}(j)\right|^{2} \delta_{j} \phi_{j, k}\right),
$$

where $\bar{h}_{k}(j)$ is the $j^{\text {th }}$ element of $\overline{\mathbf{h}}_{k}$. Note that our optimization problem has a similar form to that in [17]. Although the water-filling strategy was originally used for enhancing the channel capacity, in this work we utilize it for maximizing the instantaneous received SNR. Following [17], we arrive at an initial solution for $\phi_{j, k}$ 's as $\phi_{j, k}=\xi-\frac{N_{r} \sigma_{k}^{2}}{\varepsilon_{s}\left|\bar{h}_{k}(j)\right|^{2} \delta_{j}}$. However, depending on the channel fading gains and the receiver noise variance, this solution may not satisfy the condition $\phi_{j, k} \geq 0, \forall j$ due to the constraint $\sum_{j=1}^{N_{r}} \phi_{j, k}=N_{r}$. Thus we introduce a special notation $(x)^{+}$denoting $\max (x, 0)$. Now $\phi_{j, k}$ will be

$$
\phi_{j, k}=\left(\xi-\frac{N_{r} \sigma_{k}^{2}}{\varepsilon_{s}\left|\bar{h}_{k}(j)\right|^{2} \delta_{j}}\right)^{+} .
$$

Recall that $\delta_{j}$ 's are arranged in a non-increasing order, for now, we assume $\phi_{j, k}$ 's are also arranged in the same order, $\phi_{1, k} \geq \phi_{2, k} \geq \cdots \phi_{N_{t}, k}$, as long as $\left|\bar{h}_{k}(1)\right|^{2} \geq\left|\bar{h}_{k}(2)\right|^{2} \geq \cdots \geq\left|\bar{h}_{k}\left(N_{r}\right)\right|^{2}$. Let $B_{k}$ represent the number of non-zero $\phi_{j, k}$ 's, then $\boldsymbol{\Phi}_{k}$ becomes $\boldsymbol{\Phi}_{k}=$ $\operatorname{diag}\left[\phi_{1, k}, \cdots, \phi_{B_{k}, k}, 0_{B_{k}+1, k}, \cdots, 0_{N_{r}, k}\right]$, where $B_{k} \leq N_{r}$. Next, based on the power splitting constraint, we know that $\xi$ is chosen so that

$$
\sum_{b=1}^{B_{k}} \phi_{b, k}=\sum_{b=1}^{B_{k}} \xi-\frac{N_{r} \sigma_{k}^{2}}{\varepsilon_{s}\left|\bar{h}_{k}(b)\right|^{2} \delta_{b}}=N_{r} .
$$

Inverting (16), expressing it as a function of $\xi$, and substituting into (15) we get

$$
\phi_{j, k}=\left[\frac{1}{B_{k}}+\frac{1}{B_{k}} \sum_{b=1}^{B_{k}} \frac{N_{r} \sigma_{k}^{2}}{\varepsilon_{s}\left|\bar{h}_{k}(b)\right|^{2} \delta_{b}}-\frac{N_{r} \sigma_{k}^{2}}{\varepsilon_{s}\left|\bar{h}_{k}(j)\right|^{2} \delta_{j}}\right]^{+} .
$$

Up to this point, we still need to find a value for $B_{k}$. To find the optimal value for $B_{k}$, we set $\bar{h}_{k}(j)$ and $\delta_{j}$ to $\bar{h}_{k}\left(B_{k}\right)$ and $\delta_{B_{k}}$, respectively, then test the following inequality

$$
\frac{1}{B_{k}}+\frac{1}{B_{k}} \sum_{b=1}^{B_{k}} \frac{N_{r} \sigma_{k}^{2}}{\varepsilon_{s}\left|\bar{h}_{k}(b)\right|^{2} \delta_{b}}-\frac{N_{r} \sigma_{k}^{2}}{\varepsilon_{s}\left|\bar{h}_{k}\left(B_{k}\right)\right|^{2} \delta_{B_{k}}}>1
$$

for $B_{k}=1, \cdots, N_{r}$. Thus, the optimum value for $B_{k}$ is the largest value that satisfies the inequality, and signal transmission utilizing a number of beams that is greater than $B_{k}$ will incur a loss in the potential performance gain.

Now the optimization of (13) with respect to $\boldsymbol{\Phi}_{k}$ for a given $\mathbf{h}_{k}$ and subject to a power constraint can be written as

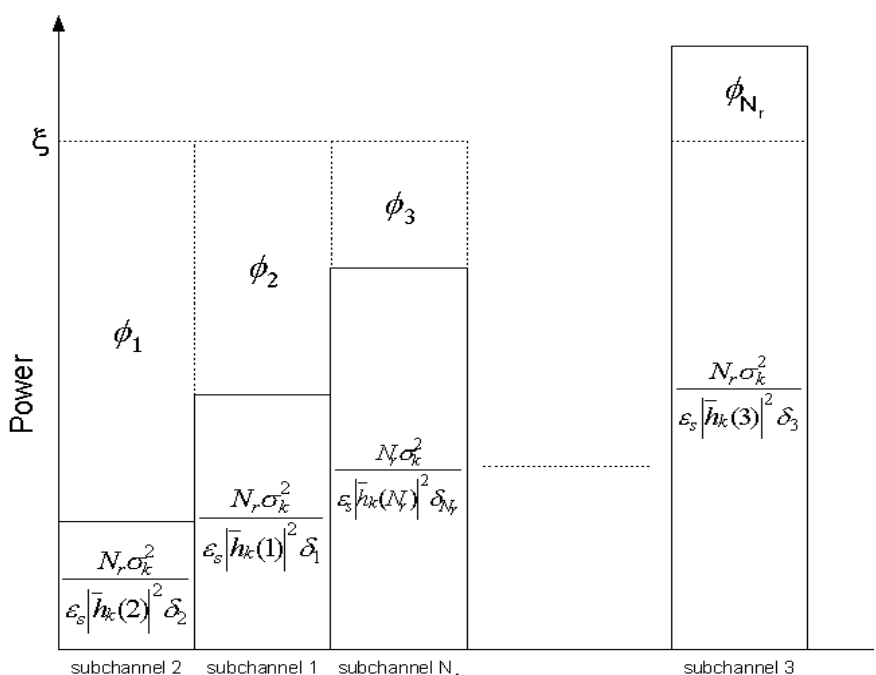

Fig. 4. Water-filling for re-ordered $N_{r}$ sub-channels.

$J \leq \prod_{b=1}^{B_{k}}\left(1+\frac{\varepsilon_{s}}{N_{r} \sigma_{k}^{2}}\left|\bar{h}_{k}(b)\right|^{2} \delta_{b} \phi_{b, k}\right)$ with equality achieved if and only if $\mathbf{W}_{k}$ is chosen as $\mathbf{W}_{k}=\mathbf{V} \boldsymbol{\Phi}_{k} \mathbf{V}^{H}$, where $\overline{\mathbf{W}}=\mathbf{V}$ and $\mathbf{U}_{k}=\boldsymbol{\Phi}_{k}$. Hence, $\mu_{j, k}=\sqrt{\phi_{j, k}}$. This shows that signal reception should be in the eigen-modes of the channel covariance matrix and effectively transforms the SIMO channel configuration into a set of $B_{k}$ parallel and independent subchannels with the $b^{\text {th }}$ subchannel having a gain of $\left|\bar{h}_{k}(b)\right|^{2} \delta_{b} \mu_{b, k}$. In case when the previous assumption $\left|\bar{h}_{k}(1)\right|^{2} \geq\left|\bar{h}_{k}(2)\right|^{2} \geq \cdots \geq\left|\bar{h}_{k}\left(N_{r}\right)\right|^{2}$ does not hold, then $\frac{N_{r} \sigma_{k}^{2}}{\varepsilon_{s}\left|\bar{h}_{k}(1)\right|^{2} \delta_{1}} \geq \frac{N_{r} \sigma_{k}^{2}}{\varepsilon_{s}\left|\bar{h}_{k}(2)\right|^{2} \delta_{2}} \geq \cdots \geq \frac{N_{r} \sigma_{k}^{2}}{\varepsilon_{s}\left|\bar{h}_{k}\left(N_{r}\right)\right|^{2} \delta_{N_{r}}}$ will not be valid. Hence, (16)-(18) will no longer be applicable since $b$ is indexing $\left|\bar{h}_{k}(j)\right|^{2}$ 's in a non-descending order. Without going to the extend of rewriting all (16)-(18) for this case, we can first re-arrange $\frac{N_{r} \sigma_{k}^{2}}{\varepsilon_{s}\left|\bar{h}_{k}(j)\right|^{2} \delta_{j}}$ 's in a non-descending order. This guarantees that $\phi_{1} \geq \phi_{2} \geq \cdots \geq \phi_{N_{r}}$. Use $b$ to index $\frac{N_{r} \sigma_{k}^{2}}{\varepsilon_{s}\left|\bar{h}_{k}(j)\right|^{2} \delta_{j}}$ 's that are less than $\xi$ (as shown in Fig. 4) instead of $\delta_{j}$ 's, such that $\phi_{j, k}$ 's and the optimum $B_{k}$ can still be found by using (17) and (18), respectively. Then signal reception is now in the directions of $B_{k}$ eigen-beams (not necessary corresponding to the first $B_{k}$ eigenvalues) that give highest instantaneous received SNR gain.

\section{Numerical Results}

In this Section we provide bit-error-rate (BER) and symbolerror-rate (SER) curves for the proposed transmission schemes in broadband frequency-selective channels. In our simulation, the following parameters and assumptions were adopted: the spatial channel correlation is modelled using the space-time channel with hyperbolically distributed scatterers in [18], $N_{c}=512, N_{t}=1$ (except for ST-OFDM, where two transmit antennas were used ), and QPSK baseband modulation is employed for both Figs. 5 and 6. In Fig. 5, we plot simulation results for systems with two receive antennas and different diversity coding schemes, i.e., OFDM with Alamouti's space- 


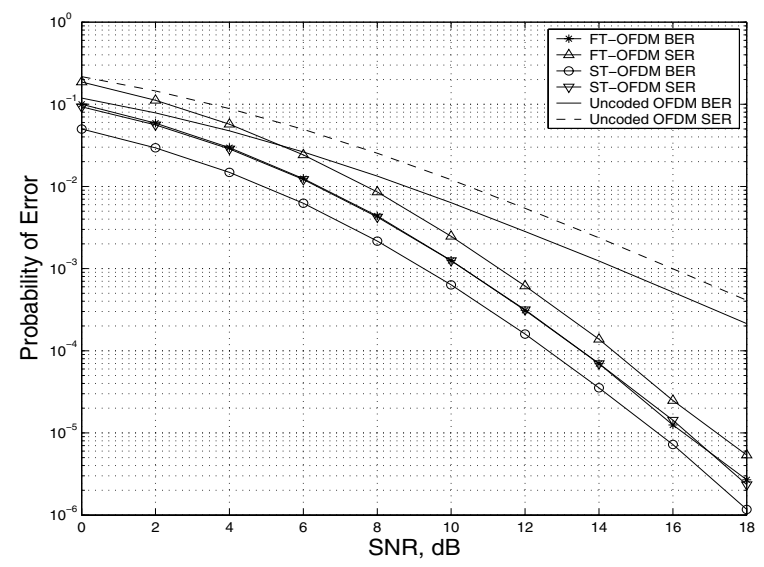

Fig. 5. BER and SER performance curves for uncoded, space-time block coded, and FT encoded OFDM systems with $N_{r}=2$.

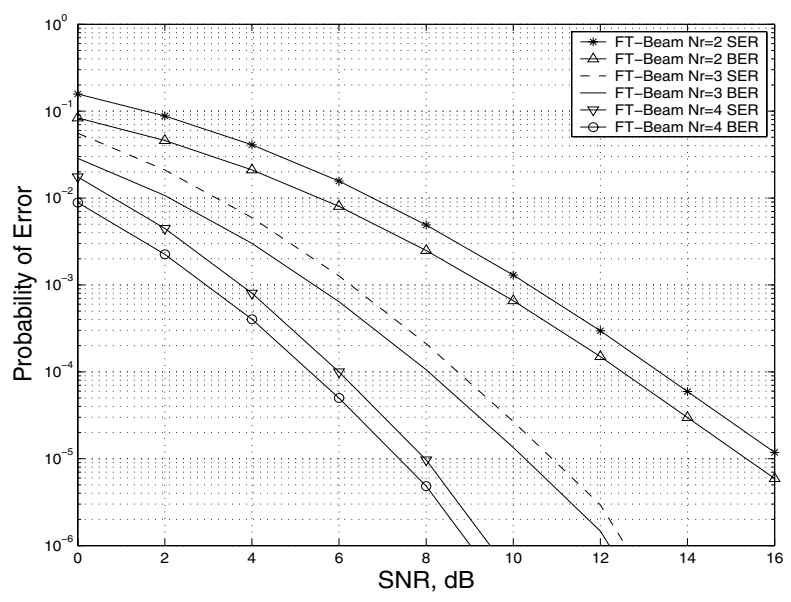

Fig. 6. BER and SER performance curves of the proposed FT-Beam transceiver scheme with different number of receive antennas.

time block code (ST-OFDM), FT encoding with Alamouti's space-time block code (FT-OFDM), and no coding (uncodedOFDM). Comparing the curves that correspond to these three schemes, it is clear that both ST-OFDM and FT-OFDM systems give significant error-rate improvement over the uncodedOFDM. Even though the FT-OFDM gives an approximately $1-\mathrm{dB}$ performance degradation with respect to the ST-OFDM scheme, it remains as an attractive scheme for hand-held terminals with only one antenna.

In Fig. 6, we showed performance curves of the proposed adaptive transceiver structure (FT-Beam) for OFDM systems with different number of receive antennas. By comparing the results for systems with $N_{r}=2$ in Fig. 6 to the results in Fig. 5 , it is clear that the error-rate performance of FT-encoded OFDM system has improved as a result of adding the receive beamforming scheme, where it is now quite comparable to STOFDM systems with two transmit antennas. Amongst the three sets of curves in Fig. 6, we see that the performance gain from adding an additional receive antenna is larger for systems with
$N_{r}=2$ than systems with $N_{r}=3$. However, it is observed that better performance curves can be obtained in channels with higher spatial correlations for FT-Beam structure.

\section{CONClusions}

An adaptive transceiver structure that combines a new diversity coding scheme and receiver beamforming for uplink SIMO-OFDM transmission is investigated. By utilizing the concept of subchannel grouping, FT coding that provides frequency diversity in the broadband wireless channel has proven to be an effective means of signal transmission for MS with a single antenna. It is shown that adaptive eigenbeamforming at the receiver (to handle uplink signals in the eigen-modes of the correlation matrix) maximizes the received SNR and improves the system error-rate performance of the FT-coded OFDM system.

\section{REFERENCES}

[1] G. J. Foschini and M. J. Gans, "On limits of wireless communications in a fading environment when using multiple antennas," Wireless Pers. Commun., vol. 6, pp. 311-335, Mar. 1998.

[2] I. E. Telatar, "Capacity of multi-antenna Gaussian channels," Eur. Trans. Tel., vol. 10, pp. 585-595, Nov./Dec. 1999.

[3] S. M. Alamouti, "A simple transmit diversity technique for wireless communications," IEEE J. Select. Areas Commun., vol. 16, pp. 14511458, Oct. 1998.

[4] V. Tarokh, H. Jafarkhani, and A. R. Calderbank, "Space-time block codes from orthogonal designs," IEEE Trans. Inform. Theory, vol. 45, pp. 1456-1467, July 1998.

[5] V. Tarokh, N. Seshadri, and A. R. Calderbank, "Space-time codes for high data rate wireless communication: Performance criterion and code construction," IEEE Trans. Inform. Theory, vol. 44, pp. 744-764, Mar. 1998.

[6] D-S. Shiu, G. J. Foschini, M. J. Gans, and J. M. Khan, "Fading correlation and its effect on the capacity of multielement antenna systems," IEEE Trans. Commun., vol. 48, pp. 502-513, Mar. 2000.

[7] H. Bolcskei and A. J. Paulraj, "Space-frequency coded broadband OFDM systems," in Proc. IEEE WCNC'00, Chicago, Sept. 2000, pp.1-6.

[8] D. Agrawal, V. Tarokh, A. Naguib, and N. Seshadri, "Space-time coded OFDM for high data-rate wireless communication over wideband channels," in Proc. IEEE VTC'98, Ottawa, May 1998, pp. 2232-2236.

[9] Z. Liu, Y. Xin, and G. B. Giannakis, "Space-time-frequency coded OFDM over frequency-selective fading channels," IEEE Trans. Signal Processing, vol. 50, pp. 2465-2476, Oct. 2002.

[10] K. H. Lin and Z. M. Hussain, "Performance of STF coded OFDM with transmit eigenbeamforming in correlated fading channels," Revised version submitted to IEEE Signal Processing Lett..

[11] K. H. Lin, Z. M. Hussain, and R. J. Harris, "Space-time OFDM with adaptive beamforming: Performance in spatially correlated channels," in Proc. IEEE TENCON, ChiangMai, Nov. 2004, pp. 617-620.

[12] A. F. Molisch, M. Z. Win, and J. H. Winters, "Space-time-frequency coding for MIMO-OFDM systems," IEEE Commun. Lett., vol. 6, pp. 370-372, Sep. 2002.

[13] E. G. Larsson and P. Stoica, Space-Time Block Coding for Wireless Communications. Cambridge, U.K.: Cambridge Univ. Press, 2003.

[14] Siemens, Channel Model for Tx Diversity Simulations using Correlated Antennas, 3GPP Document TSG-RAN WG1 \#15, R1-00-1067, Berlin, Germany, Aug. 2000.

[15] V. Tarokh, H. Jafarkhani, and A. R. Calderbank, "Space-time block coding for wireless communications: Performance results," IEEE $J$. Select. Areas in Commun., vol. 17, pp. 451-460, Mar. 1999.

[16] M. K. Simon and M.-S. Alouini, Digital Communications over Fading Channels: A Unified Approach to Performance Analysis, New York, NY: John Wiley \& Sons, 2000.

[17] T. M. Cover and J. A. Thomas, Elements of Information Theory. New York, NY: John Wiley \& Sons, 1991.

[18] S. S. Mahmoud, Z. M. Hussain, and P. O'Shea, "A space-time model for mobile radio channel with hyperbolically distributed scatterers," IEEE Antennas Wireless Propagat. Lett., vol. 1, pp. 211-214, 2002. 